\title{
EMPYEMA OR HYPOPHRENIC ABSCESS?
}

\author{
By SIR JOHN MOORE, M.D., D.P.H. DubL., F.R.C.P.I.; \\ D.Sc. Oxon. (Honoris Causâ) ;
}

Physician to the Meath Hospital and County Dublin Infirmary.

[Read in the Section of Medicine, January 19, 1906.]

THE short contribution to this discussion which I propose to make consists in notes of two cases illustrative of the difficulties which beset a differential diagnosis of empyema, or pyothorax, and hypophrenic abscess.

CASE I.-A married woman, aged thirty years, was admitted to the Meath Hospital under my care from Ranelagh Road on May 8, 1894, with a history of three weeks' illness. She had been married for fourteen years, but had never become pregnant. Menstruation had been quite regular, but was often excessive, although there was no metrorrhagia. She had been losing flesh for a year, and had also been obliged to get up at night to pass water. Six months before admission she had suffered from an attack of what was called "influenza." Although she had taken ill three weeks before admission, she did not take to bed until a week later. Her complaint was of great shortness of breath and of stabbing pain in her back and the right side of the chest. The bowels were confined, so on Sunday, May 6 (two days before admission), she took castor oil, which was followed by an excessive motion, quite black in colour.

On the evening of her admission the axillary temperature was $99.2^{\circ}$, pulse 106 , respirations 28 . The physical signs pointed to a large right empyema. Next evening temperature rose to $100.2^{\circ}$, but it fell subsequently to $96.4^{\circ}$ on the morning of May 12 . Her condition at that time was critical, and pulse and breathing were becoming more and more rapid. A free incision was, therefore, made in the ninth intercostal space in the scapular line, and one hundred and seventy (170) ounces of most offensive pus. were drawn off. The pus was deeply stained with bile, so that it 
was believed that the liver was engaged as well as the pleura. The temperature rose on the evening of the operation to $100.8^{\circ}$. A temporary intermission of fever followed, but the thermometer rose to $103.2^{\circ}$ on the evening of May 16, a septic diarrhoea set in, and the patient sank from exhaustion on the 19th.

The autopsy was made with great care by Mr. William 'Taylor, then House Surgeon to the Meath Hospital, now my colleague on the Hospital Staff. To him I am indebted for the following notes :-

"On opening the abdomen the left lobe of the liver was found to be about four times its normal size, to extend quite over to the left hypochondriac region, and to press up the lung on that side. The right lobe of the liver was nothing but an abscess sac. The gall bladder was unaffected. The neighbourhood of the pylorus showed thickening, the result of old adhesive inflammation round a gastric ulcer. There was a small opening through the diaphragm, which was adherent posteriorly as high as the fifth rib, and laterally as high as the seventh rib. There was also a moderate quantity of fluid in the right pleural cavity-about forty ounces in all.

"The right lung was greatly compressed and completely collapsed, save a small portion of the upper lobe."

In this case a diagnosis of empyema was made, whereas the suppuration was altogether below the diaphragm, constituting a hypophrenic abscess and involving the liver also. The starting point of all the mischief was probably a perforating gastric ulcer, in connection with which adhesive inflammation had occurred.

CASE II.-A farmer, aged fifty-two years, was sent to me in October, 1905, for admission to the Meath Hospital by Dr. George Peirce Ridley, J.P., Surgeon to the King's County Infirmary, and Dr. John M'Michael, of Tullamore. They considered that the patient was probably the subject of Addison's disease from the pigmentation of the tongue which was present and from the 
dark colour of the skin. The man was admitted on October 26 , 1905, and died on November 7.

The patient complained of severe pain in his stomach shooting towards the right side and upwards into his head, making him. feel dizzy. He said that the act of swallowing food was difficult, and accompanied by a sharp pain in his chest. These symptoms. dated back four months to a time when he noticed the whites of his eyes and his skin becoming yellowish.

Foul gases were frequently belched up from his stomach, the throat felt sore and swollen, and he perspired freely. Sleep. became broken. Obstinate constipation compelled him to take laxatives at frequent intervals. He lived on tea and porridge. About a week before admission cough began to trouble him, coming on in spasms or paroxysms. Whenever he tried to lieon his back or left side he was seized with intense pains. The urine became scanty, but he was frequently disturbed at night by an urgent desire to pass water. The specific gravity of the urine was 1015, but neither albumen nor sugar was present.

Of medium height, the patient weighed 9st. 616s. on admission. He said he had lost two stone within the previous two months, and was becoming terribly weak.

A faint blowing systolic murmur was heard at the apex of the heart, whence it was carried towards the back and left axilla (mitral leakage).

The abdomen was slightly full. Its wall was rigid and resisting on the right side. Intense tenderness on pressure was found overthe hepatic and gastric regions. The liver was not appreciably enlarged, but appeared to be displaced downwards. Temperaturewas generally subnormal, the ratio of pulse to respiration was. three to one. The pain, rapid wasting, progressive weakness, difficulty of swallowing, eructations of foul gas, and spasmodic cough suggested malignant disease of the csophagus-a diagnosis: which forbade the use of an œsophageal bougie, or of a stomach tube, for examination of the stomach contents. An extensivearea of dulness on percussion existed on the right side of the thorax. The dulness was absolute over the base, relative at a higher level. Vocal fremitus was absent below; present-but not to a normal degree-above. These signs suggested the presence of a considerable collection of fluid in the right pleura. So I made an 
exploration with a sterilised hypodermic syringe and drew off a clear, straw-coloured serum.

The condition of the patient now grew rapidly worse, and on November 4, 1905, his state was alarming. On that day I noted that the right side moved only slightly with respiration. The lower intercostal spaces on the same side were effaced. There was distinct fulness of the side. On pressure, some pitting was observed. From the fifth rib downwards there was absolute dulness on percussion. Over the left lung loud compensatory breathing was heard. From these signs I felt tolerably sure we had to deal with a hypophrenic abscess secondary to the œesophageal disease, and so I was not surprised when $I$ drew off a syringeful of stinking pus in the seventh intercostal space quite in front.

Mr. Taylor now took charge of the case, and incised a portion of the right tenth rib in a line with the inferior angle of the scapula, after which he evacuated an enormous collection of very foul-smelling pus, such as we would expect in an empyema arising from gangrene of the lung. A large empyema drainage tube was introduced into the cavity whence the pus had been evacuated, but the condition of the patient did not improve. He sank quickly, and died three days later.

The post-mortem showed that there was an extensive cancerous condition of the osophagus, which had ulcerated through into the posterior mediastinum, setting up a suppurative mediastinitis. From this the inflammation spread to the pleura, giving rise at first to a serous effusion upon which the infection quickly became engrafted, converting it into the purulent condition found at operation-the whole right pleural cavity had been filled with pus. The examination of the abdomen revealed nothing abnormal.

In a clinical lecture on Subdiaphragmatic Abscess, delivered at the Hospital for Consumption and Diseases of the Chest, Brompton, and published in the third volume of the fourth series of "International Clinics," 1894, Mr. Rickman J. Godlee observes that abscesses beneath the diaphragm are very difficult to differentiate from, and are very often con- 
Bined with, diseases of the chest proper. In his lecture, $\mathrm{Mr}$. Godlee describes examples of subdiaphragmatic abscess, arising not only from the liver, stomach, and the spleen, but from inflammation about the cæcum and the kidney, from rupture of the duodenum, and from caries of the ribs, and from Pott's disease of the vertebræ. He mentions the case of a lady who was sent to him on account of what was supposed to be an empyema on the right side. There was dulness in front to the level of the second rib, but the upper part of the abdomen was occupied by a large, rounded swelling, obviously connected with the liver. On enlarging a wound through which a collection of matter had been previously evacuated, Mr. Godlee found that the cavity was all below the diaphragm, and consisted of a huge suppurating hydatid cyst of the liver.

Again, he describes the case of a girl, aged twenty, who was suddenly seized with symptoms of the rupture of some hollow abdominal viscus. From this she rallied, and subsequently presented physical signs which closely simulated those of fluid in the left pleura. He was told it was an empyema, and that stinking pus had been drawn off through a canula inserted below the ninth rib in the scapular line. He repeated the exploration, and was astonished that perfectly clear and odourless serum escaped. Subsequent inquiry into the case convinced Mr. Godlee that it was one of ruptured ulcer of the stomach, and that the matter was situated between this viscus and the diaphragm.

In a third case, a girl, aged sixteen, was supposed to have empyema - a needle was inserted, but no matter was found in the pleura. On January 6, 1890, a piece of the left eighth rib was removed about the posterior axillary line, and a cavity was'opened which contained some ounces of very foul matter. The patient bore the operation well, but sank and died on the evening of the following day. At the 
time of the operation the matter was supposed to be in the pleura, but at the post-mortem it proved to be beneath the diaphragm.

Mr. Godlee reminds us that a subdiaphragmatic abscess may be accompanied by the presence of fluid in the pleura, resulting (it may be) from "sympathetic" inflammation, or from the perforation of the diaphragm by the abscess; or the same cause may give rise to the effusion of fluid in both situations.

He gives cases also in illustration of the difficulty of estimating the relation of an abscess to the diaphragm when suppuration follows caries, or necrosis of the lower ribs or rib cartilages, or of one of the lower dorsel vertebræ. It was such a case that Dr. Walter Smith submitted to this Section on December 13, 1889. His paper was published in the eighth volume of the "Transactions of the Royal Academy of Medicine in Ireland," page 19 (1890). 